\title{
"Optic Ped Scan": an alternative inexpensive technique to study plantar arches and foot pressure sites
}

\author{
A. R. Jamshidi Fard ${ }^{1} \&$ S. Jamshidi Fard ${ }^{2}$ \\ ${ }^{I}$ Department of Physiology and Biophysics, School of Medicine, \\ Arak University of Medical Sciences, Iran \\ ${ }^{2}$ McTimoney College of Chiropractic, Abingdon, UK
}

\begin{abstract}
Early diagnosis and management of foot arch abnormalities would reduce future complications. Conventional, mainly non-objective clinical examinations were not evidence based and somehow due to expert ideas with high inter rater differences. In new Pedscope we made the Optic Ped Scan (OPS), patient stand on a Resin made $5-10 \mathrm{~mm}$ Plexiglass while the image of the whole plantar surface was digitally scanned, showing the pressure sites in different colours based on a Ratio-Metric Scale. Any off-line measurement or relative pressure ratios could be easily studied. The outcome of the OPS is an image file resulting from the subject's body weight on emptying the capillaries of plantar skin which causes the colours to change. These physiological changes of plantar colour could be amplified when passing though the clear, hardly elastic form of plexiglass (Acrylic or methyl methacrylate Resin or Poly methyl 2methylpropenoate - PMMA), we prepared in Arak Petrochemical Company. We studied 2007, school age students as a pilot study of the technique in Arak schools and measured: Foot Length, Axis Length, Heel Expanse, Mid-foot Expanse, Forefoot Expanse, Arch Length (medial), Arch Depth, Hallux Distance and relative pressures of 10 defined zones.

Students had 28.15\% Flat Foot, 1.54\% Pes Cavus, 11.01\% Hallux Valgus, $0.64 \%$ Hallux Varus, $0.04 \%$ Convexus and $0.04 \%$ complex/various deformities. OPS worked properly for both diagnosis and measurements. The new technique could be several times cheaper than other techniques.
\end{abstract}

Keywords: foot arches, foot pressure sites, optic pod scan, foot deformity. 


\section{Introduction}

In 1976, Sharrard mentioned flat foot, knock knee and intoeing or metatarsus varus, as the three most common causes for parental concern when their child starts to walk [1]. Accurate diagnosis and proper management of foot arch abnormalities may reduce future musculoskeletal complications. Diagnosis, interventional treatments and estimation of the prevalence of foot deformities in screening studies need more reliable quantitative techniques than a physician's observation. Conventional, mainly non-objective clinical examinations were not evidence based and somehow due to expert ideas with high inter rater differences.

In past decades, arch exams were performed by means of a pedscope chair, a wooden chair with a thick glass on its top roof that reflects an image of plantar surface of the subject's foot to an oblique $\left(45^{\circ}\right)$ adjusted mirror set so the image could be seen from outside. With technique, the examiner was not able to measure the foot arch parameters, estimate the pressure of any particular sites or and record the findings for later comparisons but it was still helpful to show the plantar contact and weight bearing surface area of the foot with the brighter sites implying more contact pressures. The traditional solution to recording these sorts of findings, as still remembered by many middle aged colleagues, was to puff the patient's bare feet with Talc powder before asking him to stand again on a black cardboard as a printout or less durable evidence of the study.

On the other hand, new techniques such as LASER, 3D Scanners, Multi cameras and pressure platforms or sensitive mats (Pedobarographs) are much more accurate and advanced methods but they are mostly expensive and still not easily accessible in a physician's office.

This study was designed to test the hypothesis that the conventional clinical examination of the foot just by looking at a child's feet as they stand up, stand on tiptoe or dangle the foot in the air as they sit on an exam table, is insufficient for diagnosing of foot deformities.

Optic Ped Scan (OPS) or similar quantitative measurement can lead to a much better views of foot plantar surface and its actual pressure bearing areas than the conventional approach and judging based on the soft tissue mass of the medial arch.

In this paper, OPS is introduced as an alternative technique and the results of its first pilot application to detect foot deformities are presented.

\section{Methods}

In new Pedscope we made (OPS), patients stands on a Resin made of 5-10 mm Plexiglass while the image of the whole plantar surface was digitally scanned by an ordinary office type colour scanner $(\mathrm{hp}-3770)$ fixed beneath the plexiglass sheet to a computer. A 300 dpi resolution of scanning gave reasonably sharp images, showing the arches and pressure sites of the subject's foot floor in different colours based on a Ratio-Metric Scale with bright yellow indicating highly pressured areas and dark red for non pressured areas. 

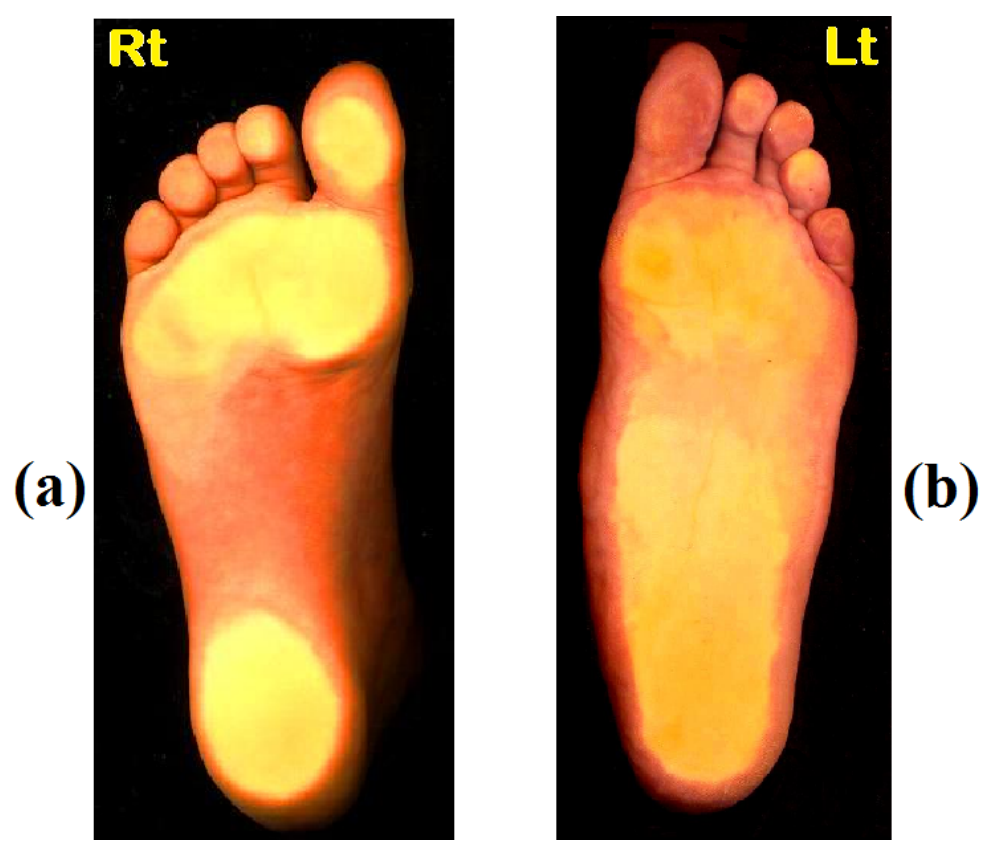

Figure 1: $\quad$ Examples of OPS layouts. (a) Pes Cavus and (b) Flat foot.

The outcome of the OPS is an image file (JPG/TIFF/ BMP) resulting from the subject's body weight on emptying the capillaries of plantar skin which turns the pressured sites into a yellowish colour while the other areas with lower weight bearing ratios are pink-reddish.

These physiological changes of plantar colour could be amplified when passing though the clear, hardly elastic form of plexiglass (Acrylic or methyl methacrylate Resin or Poly methyl 2-methylpropenoate - PMMA) which was prepared after several pilot studies (Figures 1 and 2). We optimized the aimed optical quality of this thermoplastic PMMA by reducing its stage of "Rubber Toughening" at Arak Petrochemical Co., R\&D Unit (www.arpc.ir) in Arak, Iran.

The colour density of any particular area of the image could still be a rational representative of the pressure applied or a percentage of $100 \%$ of subject's body weight if standing on one foot. In this way, all three anatomic and functional arches of the foot [2] could be scanned and sent to a computer. Any off-line measurements or calculating relative pressure ratios of each part of the achieved footprint could be easily studied.

To examine the application of the new technique and optimize the proper thickness of the PMMA sheet for a different range of subject's weight, we studied 2007, 12-13 year old Students in Level 5 of primary schools in Arak. 874 girls and 1133 boys participated in a pilot study of the technique. Each subject was scanned three times while standing on one foot (right and then left foot) and a normal double feet standing. All scanned images of each subject were saved for later analysis. 


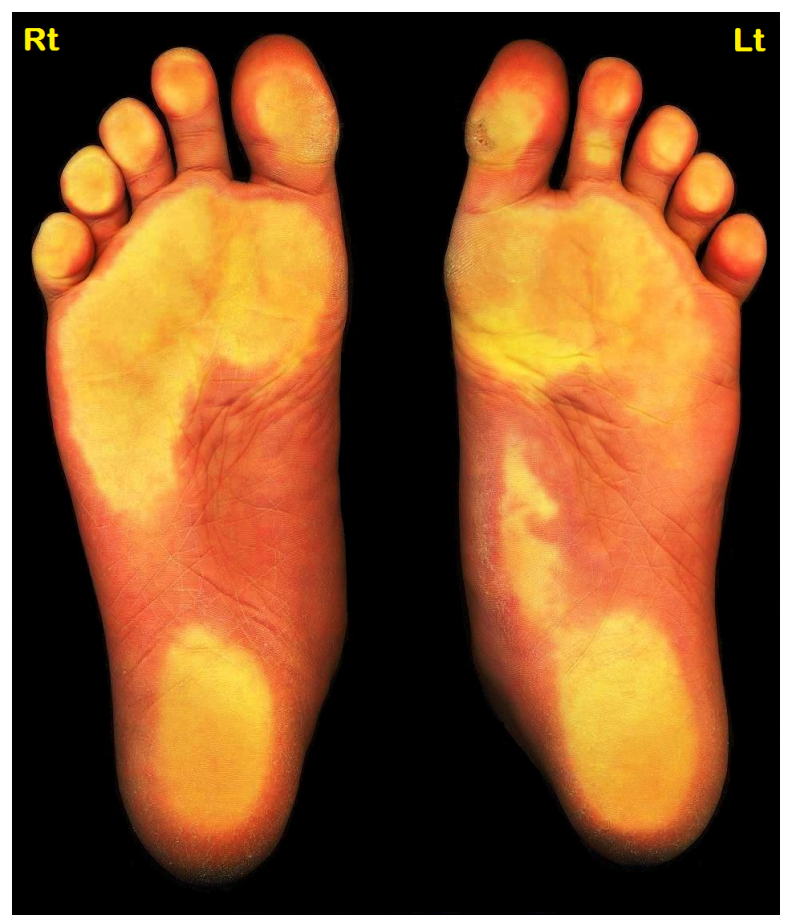

Figure 2: Examples of OPS layouts. Complex deformities in a 12 y.o. girl.

Recorded files of subjects of this technique can be opened by any Windows compatible painting or graphics software and all intended 2 dimensional measurements could be available, using the ruler of the screen in Inches, Centimetres, Millimetres or even Pixels. We used Corel Photo-Paint (CorelDraw 5, Corel Corporation, Canada) facilities for all measurements.

Most of the commercial graphics software also has an 'Equalize' filter which can redistribute shades of colours. 'Equalize' makes the darkest colour black and the lightest colour white and stretches the rest of the colours in between. We used this software to equalize the outcome image of OPS first to improve its appearance before measuring the foot plantar parameters. The Equalize filter was not used before the relative pressure measurements were taken.

The following parameters of footprints were measured: Foot Length (from heel to toe tip), Axis Length (from heel to second toe tip), Heel Expanse, Midfoot Expanse (Foot expanse at mid-arch level), Forefoot Expanse (Foot expanse at the Metatarsal heads level), Arch Length (medial bow or first ray), Arch Depth (from soft tissue line to weight borne medial border), Hallux Distance (from toe soft tissue border to main Axis) (Figure 3).

For the evaluation of the pressure distribution of the foot, each footprint obtained from OPS was divided into ten areas as functional columns of the weight bearing (Figure 4). These were the first metatarsal head, second and third 


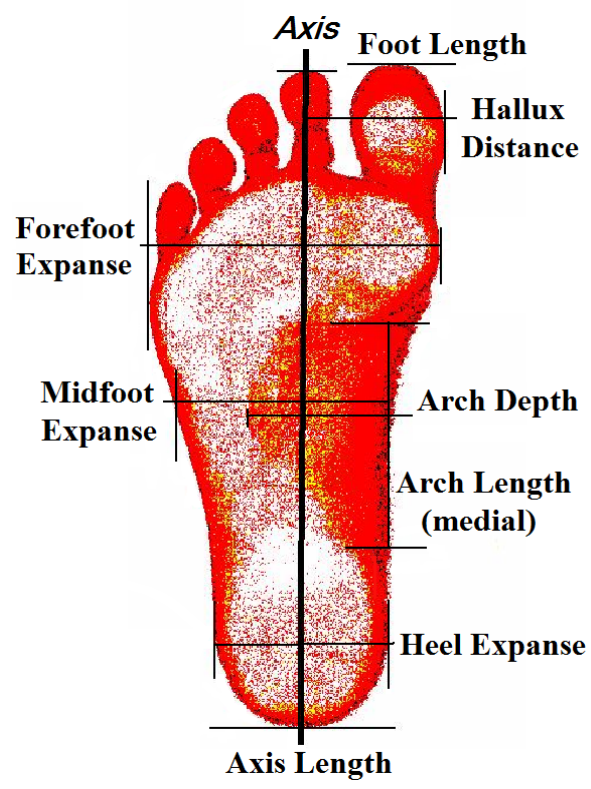

Figure 3: Foot parameters which can be measured exactly in each OPS layout.

metatarsal heads, fourth and fifth metatarsal heads and their corresponding toes in forefoot. The other areas of loading were the heel with two medial and lateral parts, the mid-foot, and the area beneath the navicular bone as the origin of the medial arch first ray $[3,4]$.

Almost all graphics software has the ability to measure a grey scale in its edit/ make colour toolbar (RGB indicator flyout) which can scale a grey colour between white (255) and black (0) relatively. To measure the relative pressure of 10 divided zones, each footprint image was first converted from colour to grey scale $(B \& W)$, then the mean relative whiteness (load) of each zone was measured, assuming the total borne weight as $100 \%$ (Figure 4).

The relative pressure measurements, even for each particular zone, could not be pooled to calculate normative data. These measurements could vary greatly because of multiple variables and the relative pressures should be studied in each subject separately.

\section{Results}

$28.15 \%$ of students (boys $34.8 \%$ and girls $22.9 \%$ ) had Flat feet based on clinical observation while based on OPS results and Denis classification only $19.8 \%$ did (boys $21.7 \%$ and girls $17.5 \%$ ). In this type of foot deformity clinical observation over estimated by $8.3 \%$ over the actual prevalence. OPS files also showed $1.54 \%$ Pes Cavus (boys 1.05\% and girls 2.17\%), 11.01\% Hallux Valgus (boys $5.82 \%$ and girls $17.73 \%$ ), $0.64 \%$ Hallux Varus (boys $0.52 \%$ and girls $0.8 \%$ ), 

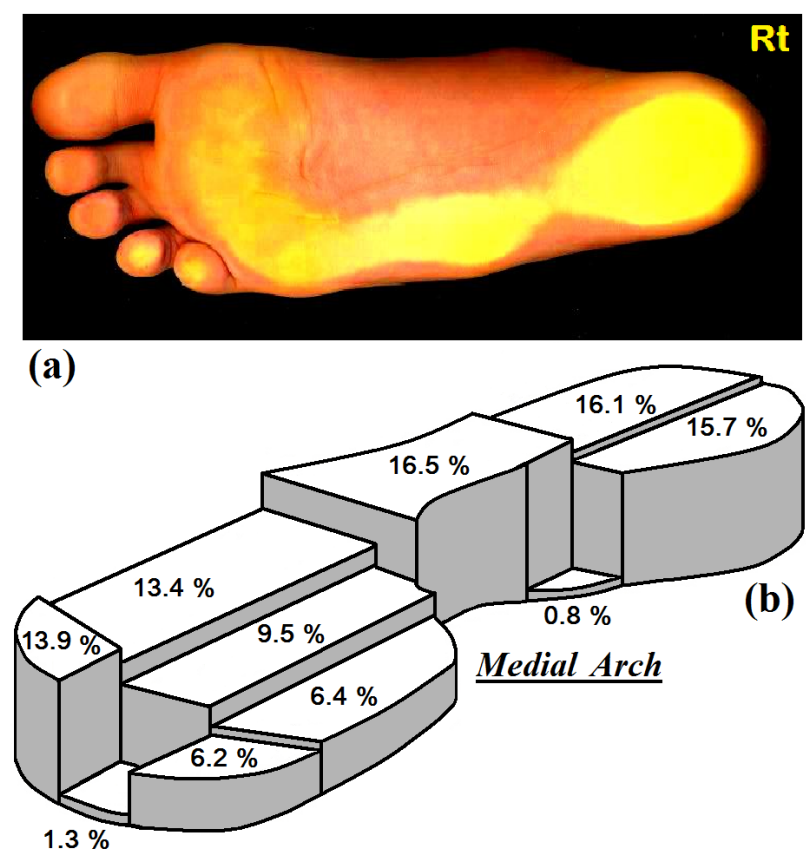

Figure 4: (a) OPS of right foot in a 12 y.o. female student with Bow legs and its abnormal relative pressure distributions on lateral border as a second heel and (b) shows its relative pressure of ten plantar zones, in schematic bars as percentage of $100 \%$ borne weight when standing on her right foot.

0.04\% Convexus (1 girl) and 0.04\% complex/various deformities (1 girl). 222 subjects had two types of deformities, therefore a sum of $28.3 \%$ of studied students were diagnosed with abnormal feet.

Foot Length, Axis Length, Heel Expanse, Mid-foot Expanse, Forefoot Expanse, Arch Length, Arch Depth, and Hallux Distance measures of each foot are summarized in Table 1. Between the two ganders, some measured parameters such as Foot Length, Axis Length, Heel Expanse, were significantly different in girls $(\mathrm{P}<0.05)$.

As an example of relative pressure measurements, the results of an abnormal weight distribution in right foot of one subject are presented in Figure 4. She was one of the participant students with bow legs and normal feet apparently.

Comparing to LASER, 3D Scanners, Multi cameras and Pedobarographs price lists of several companies with OPS set, the new technique coasts significantly less, at almost twice the price of a simple A4 colour scanner. The installed scanner software produces the image in any desired Windows format and so additional software is not necessary for this technique.

Furthermore other multi purpose graphics software available on an office computer could also be used for OPS. 
Table 1: $\quad$ The mean of measured parameters of all subjects.

\begin{tabular}{|l|c|c|}
\hline $\begin{array}{c}\text { Foot Parameters } \\
n=2007 \\
\text { (874 girls, 1133 boys) }\end{array}$ & $\begin{array}{c}\text { Right Foot } \\
n=2007 \\
(\text { Mean } \pm S D)-C m\end{array}$ & $\begin{array}{c}\text { Left Foot } \\
n=2007 \\
(\text { Mean } \pm S D)-C m\end{array}$ \\
\hline Foot Length & $22.56 \pm 1.02$ & $22.46 \pm 1.06$ \\
\hline Axis Length & $21.85 \pm 1.13$ & $21.76 \pm 1.03$ \\
\hline Heel Expanse & $5.3 \pm 0.09$ & $5.29 \pm 0.07$ \\
\hline Mid-foot Expanse & $6.66 \pm 0.1$ & $6.66 \pm 0.08$ \\
\hline Forefoot Expanse & $8.13 \pm 1.01$ & $8.15 \pm 0.93$ \\
\hline Arch Length & $5.85 \pm 0.89$ & $5.85 \pm 0.96$ \\
\hline Arch Depth & $2.42 \pm 0.66$ & $2.8 \pm 1.03$ \\
\hline Hallux Distance & $3.24 \pm 0.99$ & $3.28 \pm 1.12$ \\
\hline
\end{tabular}

\section{Discussion}

An OPS footprint with flatfoot could be classified according to Denis [5] into three grades of severity: grade 1 in which the support of the lateral edge of the foot is half of that of the metatarsal contact area (almost Mid-foot Expanse $=1 / 2$ of Forefoot Expanse); grade 2 in which the weight bearing areas of the central zone and forefoot are equal; and grade 3 in which the support in Mid- foot or central zone is greater than the width of the metatarsal support area. This classification could be described by OPS measurement methods.

Pfeiffer et al. [6], reported a prevalence of $44 \%$ in a group of 835 (411 girls and 424 boys) children of 3 to 6 years old (52\% in boys and 36\% in girls). They used a three-dimensional laser surface scanner with the subjects in a standing position. García-Rodríguez et al. [9] measured the prevalence of flat feet in 1181 school age children of Malaga in Spain as $2.7 \%$ which was the prevalence of stage 2 and 3 in Denis classification. 2.7\% of students were diagnosed to have flat feet based on plantar footprints.

Children with first degree flat foot could be considered abnormal based on clinical observation while looking at their footprints usually shows no enhancement of contact area at the central zone. These incomplete arches could be considered as evolutionary foot problems without pathological significance $[7,8]$. Prevalence of flat foot in OPS results in our subjects were closer to $12.3 \%$

Although this was mentioned by Denis, other reported a prevalence several times higher than this figure $[9,10]$.

The critical age for development of the plantar arch is 6 years [11]. Consequently, if the sampled population of such studies cover this age, the prevalence of flat feet will overestimate the problem. Infants are born with flat feet, and the main medial arch develops gradually by weight bearing during the first decade of life $[12,13]$. Most flat feet conditions correct themselves 
spontaneously at the age of 6 or more and a retarded development of the foot medial arch is reported in boys [6].

Significant differences in prevalence of flat foot is also found in overweight, obese, and normal-weight children and boys showed a significantly greater tendency for flat foot than girls [6]. It seems that different variables such as age, weight and gender may influence the prevalence rates when the subject's feet are examined in static, mainly standing positions. In addition to different parameters affecting the results of prevalence of flat foot in population screening studies, data based on clinical observations may be over estimated unless an objective technique such as OPS is adopted.

The technique of OPS shows beneath the foot and its weight borne contact surface and may reduce the examiner's bias in evaluation of children's feet.

It is worth mentioning that, in our normal subjects pressure distribution patterns of feet did not differ significantly between males or females and the right or left feet when standing on one foot alone but this pattern varied greatly between subjects when the OPS was performed in double feet weight bearing. Similar results were also reported with different techniques [3].

\section{Conclusions}

We conclude that OPS can be used sufficiently for both screening studies, diagnosis and measurements of the foot plantar parameters and relative pressure sites, but due to different types, sizes and contact areas, different weights, reciprocal weight distribution and complex structure of the foot, it is difficult to draw the line between normal and abnormal feet. The pattern of pressure distribution in each particular area may be studied before and after different interventions or compared for the clinical diagnosis of the abnormal cases but it is not possible to justify a normative data even as a percentage of borne weight.

The actual prevalence rate of foot deformities are higher than appears in literature in the region and society / school authorities involved with child health care should reconsider the foot plantar deformities of students before they start their more active future life.

\section{References}

[1] Sharrard, WJW., Intoeing and flat foot. British Medical Journal, 1, pp. 888-889, 1976.

[2] Soames, RW., Appendicular skeleton. Gray's anatomy: the anatomical basis of medicine and surgery. 38th ed., ed. Williams, PL., Bannister, LH., Berry, MM., Collins, P., Dyson, M., Dussek, JE. et al. Churchill Livingstone: New York, pp. 733-4, 1995.

[3] Kanatli, U., Yetkin, H., Simsek, A., Ozturk, AM., Esen, E., Besli, K., Pressure distribution patterns under the metatarsal heads in healthy individuals. Acta Orthop Traumatol Turc, 42(1), pp. 6-30, 2008.

[4] Myerson, MS., The diagnosis and treatment of injury to the tarsometatarsal joint complex. J. Bone Joint Surg [Br], 81, pp. 756-63, 1999. 
[5] Denis, A. Pied plat valgus statique. Encyclopedie Medico-Chirurgicale Appareil Locomoteur. Paris, France: Editions Techniques; 1974.

[6] Pfeiffer, M., Kotz, R., Ledl, T., Hauser, G. \& Sluga, M., Prevalence of Flat Foot in Preschool-Aged Children. Pediatrics, 118, pp. 634-639, 2006.

[7] Meary, R., Symposium sur le pied plant. Ann Orthop Ouest. 1, pp. 55-71, 1969.

[8] Stewart, SF., Human gait the human foot: an ethological study of flatfoot. Clinical Orthop Res. 70, pp. 111-123, 1970.

[9] García-Rodríguez, A., Martín-Jiménez, F., Carnero-Varo, M., GómezGracia, E., Gómez-Aracena, J., Fernández-Crehuet, J., Flexible flat feet in children: a real problem? Pediatrics. Jun; 103(6), e84. 1999.

[10] Gil, A., Estudio epidemiolo'gico del pie plano en escolares. Acta Pediátrica Española. 49(10), pp. 667-670, 1992.

[11] Rose, GK., Flat feet in children. Br Med J. 301, pp. 1330-1331, 1990.

[12] Volpon, JB., Footprint analysis during the growth period. J. Pediatr Orthop. 14, pp. 83-85, 1994.

[13] Forriol, F., Pascual, J., Footprint analysis between three and seventeen years of age. Foot Ankle. 11, 101-104, 1990. 